\title{
Coexistence of a Novel Medium Access Control Protocol for Wireless Ad Hoc Networks and the IEEE 802.11
}

\author{
J. Alonso-Zárate and Ch. Verikoukis \\ Centre Tecnològic de Telecomunicacions de Catalunya (CTTC) \\ Castelldefels (Barcelona, Spain) \\ \{jalonso, cveri\}@cttc.es
}

\author{
E. Kartsakli and L. Alonso \\ Dept. of Signal Theory and Communications (TSC) \\ Universitat Politècnica de Catalunya (UPC) \\ Castelldefels (Barcelona, Spain) \\ \{ellik, luisg\}@tsc.upc.edu
}

\begin{abstract}
We describe in this paper a methodology to facilitate the coexistence of the Distributed Queuing Medium Access Control (MAC) protocol for Ad hoc Networks (DQMAN) with the IEEE 802.11 Standard MAC protocol. By using dual terminals which can operate with the two MAC protocols, it is possible to run a hybrid network with legacy and novel terminals. In addition, it is possible to enable the intercommunication between all the terminals of the network. Computer simulations have been carried out to assess the performance and the feasibility of such dual operation. The main conclusion of the study is that the use of dual terminals allows the integration of DQMAN within legacy networks without degrading the performance of IEEE 802.11 terminals. In addition, the mechanism presented in this paper can be used to enable the coexistence of any cluster-based distributed MAC protocol within IEEE 802.11 networks.
\end{abstract}

\section{INTRODUCTION}

Distributed wireless ad hoc and sensor networks have been intensively studied over the past years. They are composed of a set of wireless terminals (also referred to as stations or nodes) which are able to establish a reliable communication without the presence of previously deployed infrastructure. In order to make this communication efficient and to attain the most of the radio channel, adequate medium access control (MAC) protocols are required. These protocols define the set of rules that terminals must follow in order to decide when and how they are allowed to get access to the shared medium.

In 1999, the IEEE 802.11 task group released a Standard for Wireless Local Area Networks (WLANs) which specifies the PHY and MAC layers [1]. Since then, different amendments have been done to the standard. Most of the changes have been focused on modifying the PHY layer by incorporating more advanced coding schemes, enabling the use of multiple antennas, or considering multi-hop communications either between users or access points, among others. However, the MAC protocol has remained almost unaltered across newer versions of the standard. As an example, only slight modifications were presented in the 802.11e to allow for

This work was partially funded by the Research Projects NEWCOM++ (ICT-216715), R2D2 (CP6-013), CENTENO (TEC2008-06817-C0202), PERSEO (TEC2006-10459/TCM), COOLNESS (218163-FP7-PEOPLE2007-3-1-IAPP) and by Generalitat de Catalunya (2009-SGR-940). soft service differentiation (Quality of Service) by changing the duration of the Clear Channel Assessment (CCA) and modifying the size of the contention window according to the definition of different Access Categories (ACs) [2]. The fact that the MAC layer has not been substantially modified has motivated a significant number of researchers to design novel MAC protocols that are able to overcome the wellknown limits of the IEEE 802.11 [3]. As a consequence, there are a vast number of papers in the literature proposing innovative non-backward compatible MAC protocols which improve the fairness, the energy consumption, the average transmission delay, or the network throughput by combining different techniques, in many cases resorting to cross-layer design. Unfortunately, it is not realistic to believe that already deployed equipment will be drastically replaced by any new brand MAC protocol regardless of the high performance it can attain. Therefore, it is necessary to assume that any innovative solution must be able to coexist and to intercommunicate (or interoperate) with existing commercial equipment. This is the key motivation for the work presented in this paper.

More specifically, the main contribution of this paper is the design and performance evaluation of a technique to facilitate the coexistence and intercommunication of legacy IEEE 802.11 terminals executing the Distributed Coordination Function (DCF) with new terminals executing the Distributed Queuing MAC Protocol for Ad hoc Networks (DQMAN) presented in [4]. DQMAN is the extension of the infrastructurebased high-performance DQCA protocol presented in [5] to operate in networks without infrastructure. DQMAN combines a passive, spontaneous and dynamic clustering algorithm with the execution of a near-optimum infrastructure-based MAC protocol locally within each cluster. Both analysis and simulation-based evaluation of DQMAN presented in [4] show that DQMAN can effectively overcome the performance limits of the IEEE 802.11 [2].

The coexistence between the legacy IEEE 802.11 MAC protocol and DQMAN has never been tackled before in the literature. In addition, the technique herein presented could be easily extended to any other cluster-based MAC protocol for wireless ad hoc networks. 
The remainder of the paper is organized as follows. A brief overview of the IEEE 802.11 MAC protocol is presented in Section II. The DQMAN protocol is revised in Section III. Section IV constitutes the main contribution of the paper where the coexistence and intercommunication mechanisms are described. Computer simulations to assess the feasibility of the coexistence mechanism are presented in Section V. Finally, Section VI concludes the paper and gives some final remarks.

\section{IEEE 802.11 MAC Protocol Overview}

The DCF defined in the standard for WLANs is based on a variation of the Carrier Sensing Multiple Access (CSMA) protocol with Collision Avoidance (CSMA/CA). The reader is referred to [1] for a comprehensive description of the protocol. However, for the sake of understanding of the paper, we present in this section a very brief overview of it. An example of operation of the protocol is illustrated in Fig. 1.

Whenever a user has data ready to be transmitted, it performs the CCA function to decide whether the channel is idle or busy. If the channel is idle for a Distributed Inter Frame Space (DIFS) period, then the user initiates the transmission. Otherwise, it executes a Binary Exponential Backoff (BEB) algorithm by which it sets a backoff counter to an integer random value within a contention window defined in the interval of $[0, C W]$ with $C W=C W_{\min }$ the minimum size of this contention window. This counter will be decremented as long as the channel is idle. Whenever the counter gets to zero, the user attempts to transmit. In the case of failure (collision or transmission error), the $C W$ is doubled up, up to a given maximum value $C W_{m}=2{ }^{m} C W_{\min }=C W_{M A X}$, and the backoff counter is reset to a random value within the interval $\left[0, C W_{i}\right]$, with $i=1, \ldots, m$. Note that $m$ is the maximum backoff stage. Therefore, the value of the $C W$ can be summarized for any backoff stage $i$ as

$$
C W_{i}=\min \left\{2^{i} C W_{\min }, C W_{M A X}\right\} .
$$

Any packet is discarded after $m^{\prime}$ failed transmission attempts and the $C W$ is reset to the initial value $C W_{\min }$ in order to process the next packet.

Two access methods are defined in the IEEE 802.11 standard, namely, the basic access and the collision avoidance (CA) access. In the former method, data packets are immediately transmitted when trying to seize the channel, while in the later method a handshake (Request-to-Send (RTS)-Clearto-Send (CTS)) between source and destination is established before initiating the actual data transmission to combat the hidden terminal problem. Independently of the access method,

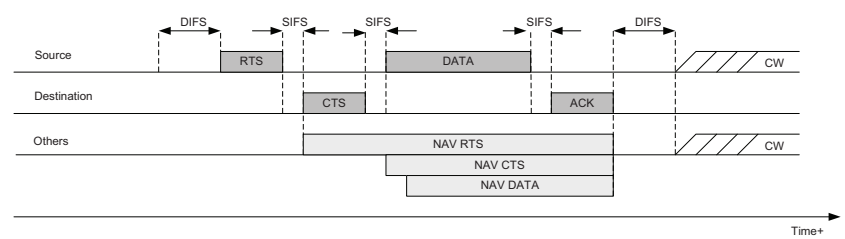

Fig. 1. Example: IEEE 802.11 MAC Protocol upon the correct reception of a data packet, the destination station sends back an ACK packet after waiting for a Short Inter Frame Spaces (SIFS). This SIFS is necessary to compensate for propagation delays and radio transceivers turnaround times to switch from receiving to transmitting mode. It is worth noting that due to the fact that a SIFS is shorter than a DIFS, acknowledgments have higher priority than data transmissions.

An important feature of the standard is the Virtual Carrier Sensing (VCS) mechanism; those stations not involved in an ongoing transmission avoid attempting to get access to the channel for the time the ongoing transmission is expected to last and regardless of the physical channel carrier sensing. Towards this end, users update a Network Allocation Vector (NAV) with the duration field attached to the header of all transmitted packets indicating the time that the channel has to be reserved to avoid a collision (see Fig. 1).

\section{DQMAN OVERVIEW}

Due to space constraints, it is not possible to fully describe the operation of DQMAN in this paper. However, and for the sake of understanding of the mechanisms presented in this work, an overview of the keys of the protocol is presented in this section. The reader is referred to [4] for a comprehensive description and theoretical analysis of DQMAN.

\section{A. Clustering Algorithm}

When DQMAN is applied, stations get self-organized into spontaneous and temporary master-slave clusters. Despite the cluster structure, communications are performed in a peerto-peer fashion between any pair of source and destination stations.

The clustering algorithm of DQMAN is based on CSMA and following an operation similar to that of DCF. Three modes of operation are defined, namely, idle, master, and slave. Any station must be able to operate in any of these states and switch from one to another whenever necessary. The protocol works as follows. Any idle station with data ready to transmit executes a CCA function, i.e., it senses the channel in seek for an already existing master station. If a master is found, then the station becomes slave and implicitly joins the cluster of the master. Otherwise, it sets itself to master mode and periodically transmits a control beacon. This beacon is necessary to broadcast some control information and to enable idle stations to associate to the cluster. A station operates in master node for as long as there is data activity within its cluster. Whenever the data buffers of the master are empty and there are no new access requests for the channel, the station operating in master mode reverts to idle mode. Therefore, the cluster topology varies as a function of the traffic load of the network. In order to avoid static cluster settings due to the heavy traffic conditions, a Master Time Out (MTO) mechanism is considered in DQMAN. Whenever a station is set to master mode, it initiates a MTO counter to a deterministic integer value. After the transmission of each periodic control beacon, as described before, the MTO counter is decremented by one. Upon expiration of the counter (when 


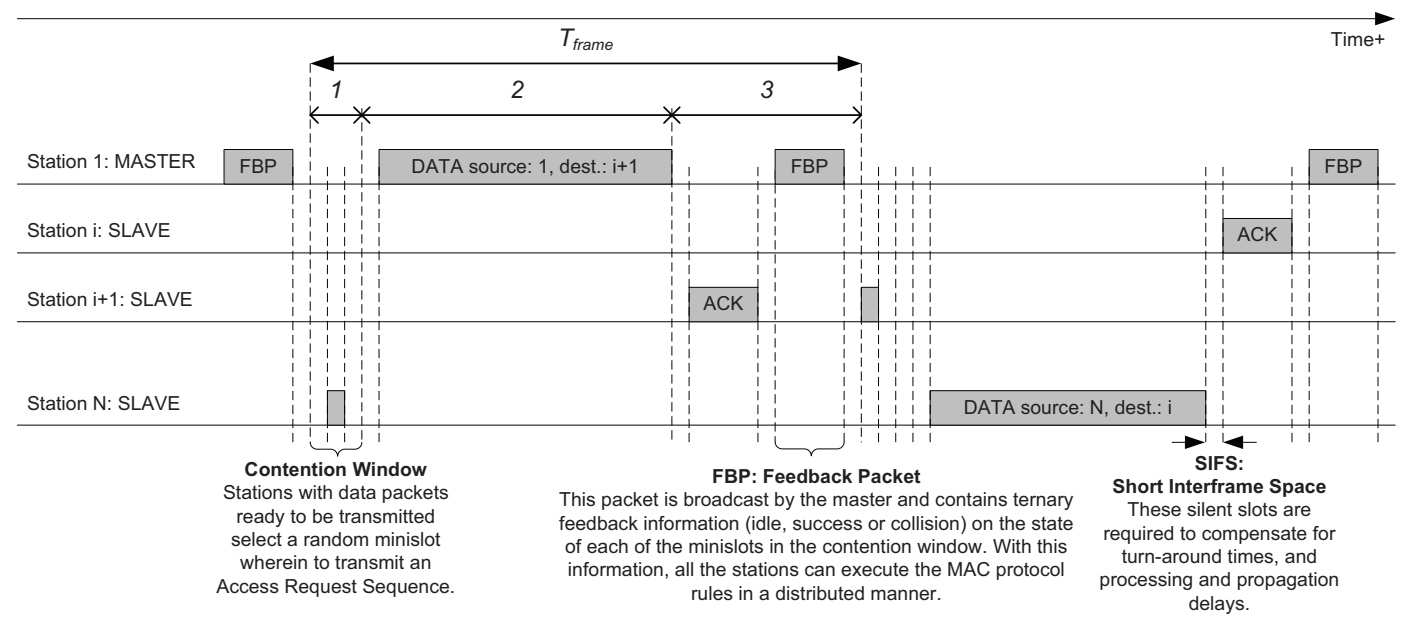

Fig. 2. DQMAN Frame Structure

the counter hits zero), the master station reverts to idle mode regardless of the data activity within its cluster. All the slave stations which stop receiving the control beacon from the master become idle and the cluster is broken.

\section{B. MAC Protocol}

The periodic control beacon broadcast by the master gets the form of a control packet, named Feedback Packet (FBP). The FBP defines a time-frame MAC structure, as depicted in Fig. 2. Slave stations can get synchronized with the master station by detecting the preamble attached to the FBP. The MAC frame is divided into 3 parts of different duration:

1) The first part is further divided into an integer number of access minislots wherein those stations with data ready to be transmitted send a channel Access Request Sequence (ARS) in the form of a coded sequence of chips.

2) The second part is devoted to data transmissions.

3) The third part is devoted to the exchange of control information. This last part consists of a slot reserved for the transmission of ACK packets from any destination station upon reception of a data packet and another slot reserved for the broadcast of the FBP by the master station containing the minimum control information required for the MAC operation [5].

In addition, a SIFS is left between each part of the frame in order to tolerate non-negligible propagation and processing delays, as well as turnaround times to switch the RF from receiving to transmitting mode.

\section{COEXISTENCE AND InTERCOMMUNiCATION}

\section{A. Scenario}

We consider a wireless ad hoc network formed by a number of stations that can be split into two groups:

1) Standard stations which can only operate with the DCF of the IEEE 802.11 MAC protocol. These stations are not aware of the presence of other kind of stations and do not know the rules of DQMAN.
2) Dual stations which can operate with both DCF and DQMAN. By default, these stations execute the rules of DCF with collision avoidance access method, i.e., with RTS-CTS handshake. It is worth noting that this can be easily imposed in current commercial devices by setting the RTS threshold to 0 bytes, so that all packets are transmitted with RTS-CTS [1].

\section{B. Coexistence}

Any dual station advertises its dual capability to its neighbors by setting to "1" a dual flag within any transmitted RTS or CTS packet. This flag is ignored by standard stations. Towards this end, it is worth noting that RTS and CTS control packets do not use some of the bits within their 2 byte-length Frame Control (FC) field. As an example, either bit B8 or B9 of the FC field could be used as the dual flag [1]. These two bits are only used for data packets and within the context of infrastructure-based networks, and remain unused otherwise.

Upon the reception of an RTS packet, a dual station crosschecks the value of the dual flag and acts accordingly:

1) If Dual Flag $=1$, the station initiates a DQMAN phase by setting itself to master mode and transmitting a CTS packet with the dual flag set.

2) If Dual Flag $=0$, it transmits a regular CTS packet and the regular operation of the DCF is executed.

If a DQMAN phase is initiated:

1) The master station periodically broadcasts a FBP. In addition to the control information related to the operation of DQMAN, this packet must contain the information of the duration of the MAC frame so that standard stations can update their NAV to avoid attempting to get access to the channel. However, since FBP packets cannot be decoded by standard stations, this information must be attached to all data packets, which should account for the transmission time of the FBP.

2) Dual stations get implicitly associated to the master station by entering the slave mode. 
3) The master will revert to idle mode whenever there are no more data packets ready to be transmitted among all the stations of the cluster and whenever there are no more collisions to be resolved. As explained before, the MTO mechanism protects the network from operating only in DQMAN mode and thus blocking standard stations from accessing the channel. Therefore, the value of the MTO should be set taking into account the minimum channel access guarantees for standard stations.

4) Upon disassociation from a cluster, any dual station executes a backoff. The value of the backoff counter is set using the maximum size of the contention window so that standard stations have greater probability of getting access to the channel right after a DQMAN phase has occurred.

\section{Intercommunication}

Intercommunication between standard and dual stations is implicitly enabled with the coexistence mechanism described in the previous section. Note that the exchange of data packets between two standard stations and between a standard and a DQMAN station is performed by executing the DCF. In addition, although standard stations cannot get access to the channel to transmit their data packets when a DQMAN cluster is running, they may receive data packets. This means that for the duration of a DQMAN phase, communication between standard stations is not possible, and between standard stations and dual stations only works in one direction. This is the main motivation to force the re-clustering of the network from time to time under heavy traffic conditions. Otherwise, standard stations could suffer from starvation and the channel access fairness of the system would be compromised.

\section{PERformance Evaluation}

\section{A. Description}

In order to assess the feasibility of the coexistence mechanism presented in this paper, a mixed scenario formed by a number of standard stations and a number of dual stations has been simulated with a custom-made $\mathrm{C}++$ software simulator. The complexity of the scenario made difficult its integration in the ns-2 simulator and thus a custom solution was adopted.

Three single-hop networks (all the stations are within the transmission range of each other) with a total of $N=10$ stations have been simulated:

1) Mixed Network: 5 out of the 10 stations are standard stations and the other 5 are dual stations which can execute both the DCF of the IEEE 802.11 and DQMAN.

2) 802.11 Network: the 10 stations only execute the DCF of the IEEE 802.11.

3) DQMAN Network: the 10 stations only execute DQMAN, without DCF operation.

The rest of the parameters of the simulation have been set according to the IEEE $802.11 \mathrm{~g}$ and they are summarized in Table I. The size of data packets has been set to 1500 bytes since, according to [8], this is the data packet size that better represents the data traffic of an actual WLAN.

\section{B. Results}

The throughput, defined as the total data bits transmitted per second among all the stations of the network, is depicted in Fig. 3. The main conclusion is that the coexistence mechanism works in terms of total network throughput. As it could be expected, the performance of the mixed network lies in between the performance of the DQMAN and the 802.11 networks. It is worth noting that the greater the value of the MTO, the closer the performance to that of a DQMAN network. Further, it is interesting to evaluate how the throughput is shared among the different kinds of stations in the mixed network. Towards this end, the throughput of the group of 5 standard stations and that of the group of 5 dual stations is illustrated in Fig. 4. It is interesting to see that for low traffic loads, the throughput attained by each of the two groups is identical. However, when the traffic load grows, DQMAN phases last for longer periods of time, thus decreasing the performance of IEEE 802.11 stations. The difference between the saturation throughput of the DQMAN group and the IEEE 802.11 group depends on the value of the MTO. Therefore, the selection of the MTO is an important parameter to be taken into account when deploying this kind of mixed networks. The average packet transmission delay of the stations is illustrated in Fig. 5 . The mixed network performs slightly worse than any of the two pure networks in terms of average delay. This is mainly due to the fact that for the duration of a DQMAN phase, the average access delay of IEEE 802.11 stations is increased, and so is their average packet delay. Recall that for the duration

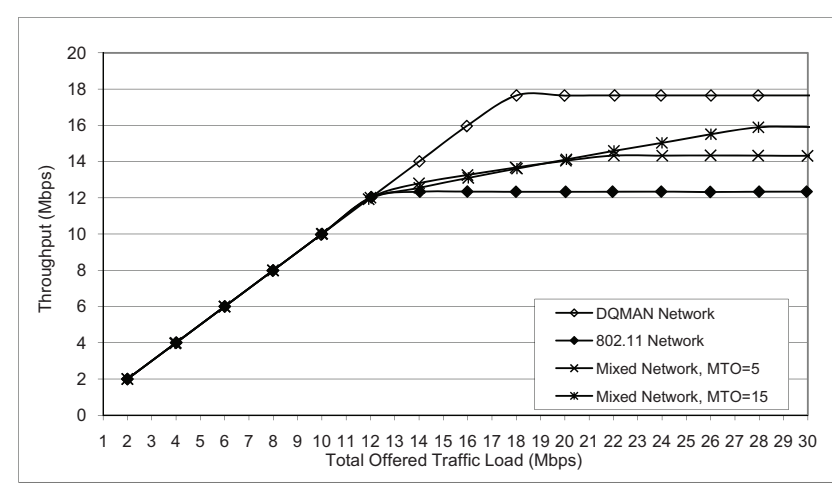

Fig. 3. Throughput (Mbps)

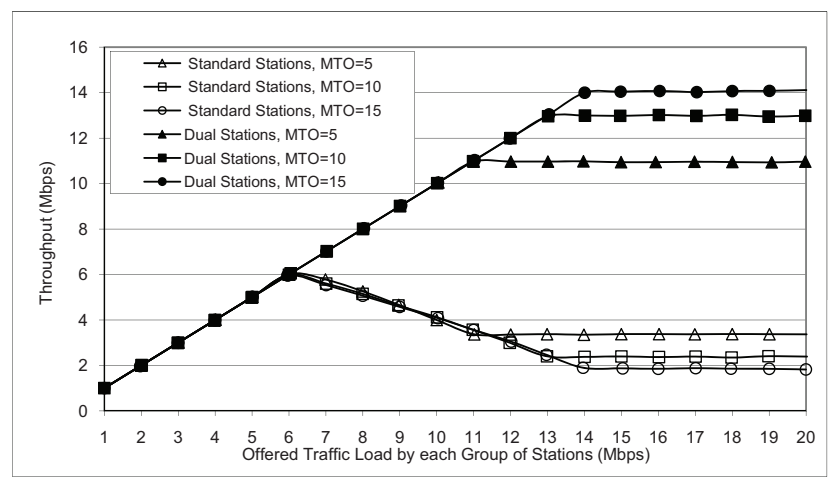

Fig. 4. Throughput Attained by Each Group of Stations 
TABLE I

Simulation PARAMEters

\begin{tabular}{|c|c|c|c|}
\hline Parameter & Value & Parameter & Value \\
\hline \hline Data Tx. Rate & $54 \mathrm{Mbps}$ & Ctrl. Tx. Rate & $6 \mathrm{Mbps}$ \\
\hline MAC header & 34 bytes & PHY preamble & $96 \mu \mathrm{s}$ \\
\hline ACK length & 14 bytes & Data packets & 1500 bytes \\
\hline \multicolumn{4}{|c|}{ IEEE 802.11} \\
\hline DIFS & $50 \mu s$ & SIFS & $10 \mu \mathrm{s}$ \\
\hline$C W_{\min }-C W_{M A X}$ & $32-64$ & SlotTime & $10 \mu \mathrm{s}$ \\
\hline RTS length & 20 bytes & CTS length & 14 bytes \\
\hline \multicolumn{4}{|c|}{ DQMAN } \\
\hline Minislots & 3 of $10 \mu s$ & ARS and SIFS & $10 \mu \mathrm{s}$ \\
\hline FPB length & 14 bytes & MTO & 100 frames \\
\hline
\end{tabular}

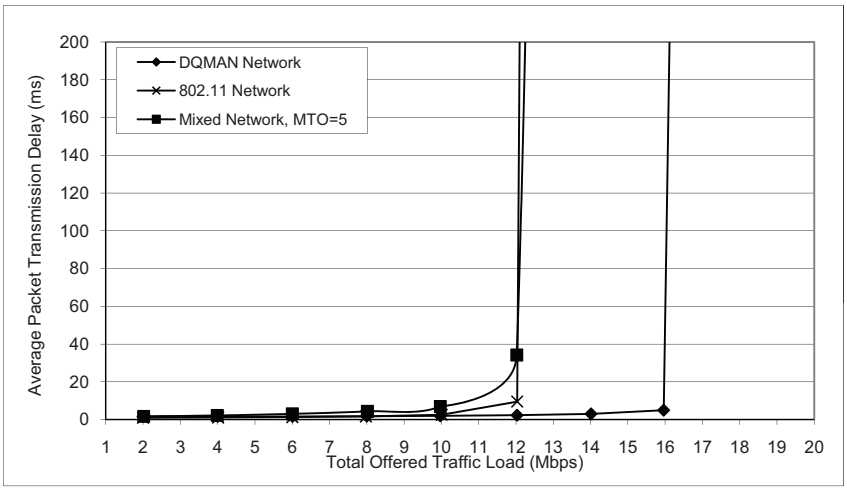

Fig. 5. Average Packet Transmission Delay (ms)

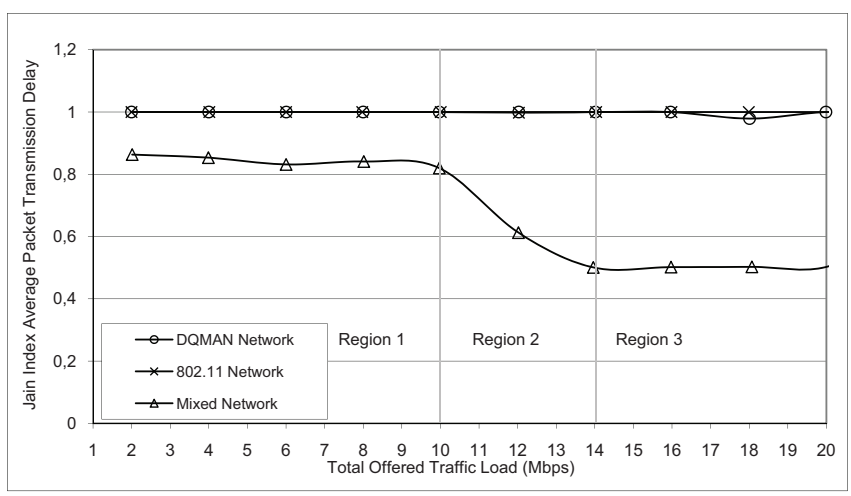

Fig. 6. Jain Index of the Average Packet Transmission Delay

of an active DQMAN phase, standard stations cannot get access to the channel to transmit their data packets. To better understand this situation, the Jain Index [7] of the average packet transmission delay in the three different scenarios is presented in Fig. 6. This parameter allows evaluating the fairness of the network in terms of average packet transmission delay. It has a value between 0 (totally unfair) and 1 (perfect fairness) and intuitively represents the fairness of the system. We denote by $T_{i}$ to the average packet transmission delay of station, and thus the Jain Index is computed as

$$
J I=\frac{\left(\sum_{N} T_{i}\right)^{2}}{N \sum_{N} T_{i}^{2}} .
$$

As it can be inferred from Fig. 6, both the IEEE 802.11 and the DQMAN networks attain, in average, long-term fairness $(J I=1)$. Therefore, all the stations attain similar performance in terms of average delay. However, as it could be expected, in the mixed network, fairness is slightly affected $(J I=0.85)$. Note that some of the stations are dual and thus attain lower delays when operating during a DQMAN phase. Under heavy traffic conditions, the fairness is decreased in a more remarkable manner. The better performance of DQMAN becomes more evident as the offered load increases. It is worth noting that due to the symmetry of the scenario where half of the users are dual and half are standard, the $J I$ tends to a value of 0.5 when the offered load is higher than $12 \mathrm{Mbps}$. Note that this lack of perfect fairness is due to the fact that half of the users can get access to the channel in a more efficient manner than the other half of the users. Therefore, this performance is caused by the inherent composition of the network, and not by the coexistence mechanism presented in this paper.

\section{Conclusions}

The coexistence and intercommunication of the IEEE 802.11 Standard MAC protocol with the high-performance DQMAN protocol for wireless ad hoc networks has been presented in this paper. By considering the use of dual terminals capable of executing the rules of the two protocols, it is possible to run a mixed network formed by standard and dual terminals simultaneously and to attain good performance figures. Computer-based simulations have been carried out to assess the feasibility of the proposed methodology. Results show that both coexistence and intercommunication between legacy and new DQMAN stations are possible. This result is very important in the light of an actual successful implementation of DQMAN. In addition, this methodology could be easily applied to any other cluster-based MAC protocol for wireless ad hoc networks.

\section{REFERENCES}

[1] IEEE Std. 802.11 WG, "Wireless LAN Medium Access Control (MAC) and Physical Layer (PHY) Specifications," 1999, Standard.

[2] B. H. Walke, S. Mangold, L. Berlemann, "IEEE 802 Wireless Systems," John Wiley \& Sons, 2006.

[3] X. Yang and J. Rosdahl, "Throughput and delay limits of IEEE 802.11," IEEE Communication Letters, vol. 6, no. 8, pp. 355-357, Aug. 2002.

[4] J. Alonso-Zárate, E. Kartsakli, Ch. Verikoukis, and L. Alonso, "Saturation throughput analysis of a passive cluster-based medium access control protocol for ad hoc wireless networks," in Proc. of the ICC 2008, May 2008.

[5] J. Alonso-Zárate, E. Kartsakli, A. Cateura, C. Verikoukis and L. Alonso, "A near-optimum cross-layered distributed queuing protocol for wireless LAN," IEEE Wireless Communication Magazine, vol. 15, no. 1, pp. 48-55, Feb. 2008

[6] IEEE Std. 802.11g, Supplement to Part 11: Wireless LAN Medium Access Control (MAC) and Physical Layer (PHY) Specifications; Further High-Speed PHY Layer Extension in the $2.4 \mathrm{GHz}$ Band, 2003.

[7] R. Jain, D. Chiu and W. Hawe, "A quantitative measure of fairness and discrimination for resource allocation in shared computer systems," DEC Research Report, TR-301, vol. 2, no. 5, pp. 1017-1028, Sep. 1984.

[8] J. Yeo, M. Youssef and A. Agrawala, "Characterizing the IEEE 802.11 traffic: the wireless side," Technical Report, Univ. of Maryland, College Park, Rep. CS-TR-4570, Mar. 2004, http://www.cs.umd.edu/Library/TRs/CS-TR-4570/CS-TR-4570.pdf. 